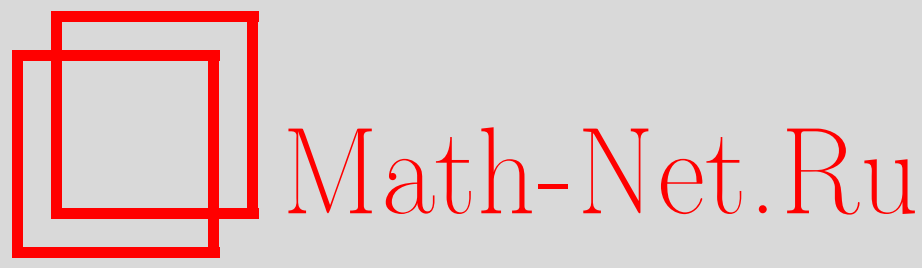

М. А. Браун, Энергия Казимира квантованного поля в среде с дисперсией и поглощением, ТМФ, 2013, том 175, номер 3, 388-397

DOI: https://doi.org/10.4213/tmf8478

Использование Общероссийского математического портала Math-Net.Ru подразумевает, что вы прочитали и согласны с пользовательским соглашением http://www . mathnet.ru/rus/agreement

Параметры загрузки:

IP : 34.227 .88 .159

26 апреля 2023 г., $12: 20: 57$

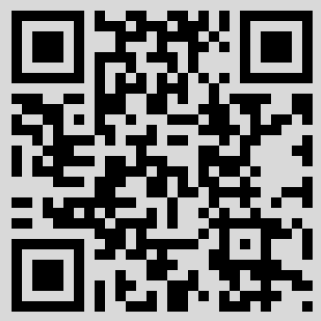




\title{
ЭНЕРГИЯ КАЗИМИРА КВАНТОВАННОГО ПОЛЯ В СРЕДЕ С ДИСПЕРСИЕЙ И ПОГЛОЩЕНИЕМ
}

\begin{abstract}
Энергия Казимира квантового поля между двумя металлическими пластинами, разделенными диэлектриком, вычислена в микроскопическом подходе, при котором диэлектрик моделируется набором осцилляторов с непрерывно распределенными частотами. Рассмотрен случай одного пространственного измерения. Сравнение вычисленных дополнительной энергии и силы, возникающей за счет взаимодействия со средой, с результатами, полученными в рамках феноменологического подхода, сходного с применением известной формулы Лифшица, показывает, что они имеют противоположные знаки.
\end{abstract}

Ключевые слова: силы Казимира, поглощение квантового поля средой, диагонализация Фано, формула Лифшица.

DOI: $10.4213 / \operatorname{tmf} 8478$

\section{1. ВВЕДЕНИЕ}

В рамках макроскопической электродинамики изучение сил Казимира в диэлектрике с дисперсией и поглощением может быть осуществлено с помощью введения стохастического шума, имитирующего диссипативное взаимодействие со средой [1], [2]. Однако последовательная трактовка требует корректного квантово-механического описания этого взаимодействия. Оно может быть осуществлено в рамках формализма, развитого в работе Хуттнера и Барнетта [3] и позднее обобщенного Филбиным [4], в котором предполагается, что среда моделируется набором осцилляторов с непрерывно распределенными частотами [5]. Такое взаимодействие приводит к поглощению поля и его исчезновению как независимой динамической переменной. Система полностью описывается в терминах эффективных осцилляторов, также имеющих непрерывно распределенные частоты. Возбуждения квантованного поля выражаются через возбуждения этих эффективных осцилляторов. Энергия Казимира в этом подходе была исследована Филбиным [6], однако в его подходе влияние среды не отделено от общей энергии Казимира в среде. Кроме того, его общие результаты трудно приложимы к простым многослойным диэлектрикам. Настоящая работа близко следует идеологии работ [3], [4]. Разница заключается в более

* Санкт-Петербургский государственный университет, Санкт-Петербург, Россия. E-mail: braun1@pobox.spbu.ru 
простой модели среды и в новой технике, допускающей четкое выделение эффектов среды и облегчающей применение к таким простым случаям, как диэлектрик между двумя металлическими пластинами. Для этого случая мы вычисляем дополнительную энергию Казимира и силу, возникающую за счет взаимодействия со средой, и сравниваем их с результатами, полученными в макроскопическом подходе с использованием техники функции Грина [7]. В обоих случаях результаты оказываются малы, но имеют противоположные знаки. На этом предварительном этапе мы ограничиваемся скалярным полем в одном пространственном измерении.

\section{2. МОДЕЛЬ. ДИАГОНАЛИЗАЦИЯ ФАНО}

В интервале пространственной переменной $0<x<a$ наша модель описывает набор дискретных оцилляторных мод, взаимодействующих с континуумом. Эта модель обобщает модель, предложенную Фано [8] для демонстрации того, как континуум расплавляет дискретную моду и таким образом осуществляет поглощение. В этом разделе мы воспроизводим реализацию идеи Фано в технике работы [3] с некоторыми изменениями.

В нашей модели лагранжиан $L(t)$ есть сумма трех слагаемых: $L=L_{0}+L_{1}+L_{\mathrm{I}}$. Здесь $L_{0}$ есть лагранжиан поля

$$
L_{0}=\frac{1}{2} \int_{0}^{a} d x\left(\dot{\phi}^{2}-(\partial \phi)^{2}-\mu^{2} \phi^{2}\right),
$$

где $\dot{\phi}$ и $\partial \phi$ обозначают производные по времени $t$ и координате $x$ соответственно.

Поглощающая среда моделируется набором осцилляторов на том же интервале координат $[0, a]$ с непрерывно распределенными частотами:

$$
L_{1}=\frac{1}{2} \int_{0}^{a} d x \int_{0}^{\infty} d \omega\left(\dot{Y}_{\omega}^{2}-\omega^{2} Y_{\omega}^{2}\right) .
$$

Наконец, $L_{\mathrm{I}}$ описывает взаимодействие между квантовым полем и средой. Следуя [3], мы выбираем его в виде

$$
L_{\mathrm{I}}=-\int_{0}^{a} d x \int d \omega v(\omega) \phi \dot{Y}_{\omega},
$$

где $v^{2}(\omega)$ - квадратично-интегрируемая функция, которая может быть аналитически продолжена на отрицательные $\omega$ как четная функция. Мы налагаем граничные условия, соответствующие металлическим пластинам:

$$
\left.\phi(x)\right|_{x=0}=\left.\phi(x)\right|_{x=a}=0,\left.\quad Y_{\omega}(x)\right|_{x=0}=\left.Y_{\omega}(x)\right|_{x=a}=0 .
$$

Для квантования мы стандартным образом вводим сопряженные переменные и налагаем канонические одновременнь́е коммутационные соотношения. В импульсном пространстве интеграл по области $[0, a]$ переходит в сумму по дискретным значениям импульса $k_{n}=\pi n / a, n=1,2, \ldots$.

Мы вводим операторы рождения и поглощения для поля $b_{n}, b^{\dagger}$ и среды $b_{n, \omega}, b_{n, \omega}^{\dagger}$ со стандартными коммутационными соотношениями. В терминах этих операторов находим полный гамильтониан

$$
H^{\text {tot }}=H_{0}+H_{1}+H_{\mathrm{I}}+e_{0}(a)+e_{1}(a)=H+e_{0}(a)+e_{1}(a),
$$


где $H_{0}$ и $H_{1}$ - гамильтонианы квантового поля и среды в нормальной форме,

$$
H_{0}=\sum_{n} \omega_{n} b_{n}^{\dagger} b_{n}, \quad H_{1}=\sum_{n} \int_{0}^{\infty} d \omega \omega b_{n, \omega}^{\dagger} b_{n, \omega}
$$

Сдвиг энергии вакуума есть сумма таких сдвигов для поля $\phi$ и среды

$$
e_{0}(a)=\frac{1}{2} \sum \omega_{n}, \quad e_{1}(a)=\frac{1}{2} \sum_{n} \int \omega d \omega .
$$

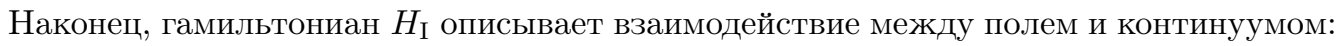

$$
H_{\mathrm{I}}=\frac{1}{2} \sum_{n} \int_{0}^{\infty} d \omega V_{n}(\omega)\left(b_{n}+b_{n}^{\dagger}\right)\left(b_{n, \omega}+b_{n, \omega}^{\dagger}\right)
$$

где $V_{n}^{2}(\omega)=\left(\omega / \omega_{n}\right) v^{2}(\omega)$.

Можно показать, что гамильтониан $H$ может быть диагонализован введением новых полевых переменных [3]

$$
B_{n, \omega}=\alpha_{0 n}(\omega) b_{n}+\beta_{0 n}(\omega) b_{n}^{\dagger}+\int_{0}^{\infty} d \omega^{\prime}\left[\alpha_{1 n}\left(\omega, \omega^{\prime}\right) b_{n, \omega^{\prime}}+\beta_{1 n}\left(\omega, \omega^{\prime}\right) b_{n, \omega^{\prime}}^{\dagger}\right],
$$

которые удовлетворяют коммутационным соотношениям

$$
\left[B_{n, \omega}, B_{n^{\prime}, \omega^{\prime}}^{\dagger}\right]=\delta_{n n^{\prime}} \delta\left(\omega-\omega^{\prime}\right), \quad\left[B_{n, \omega}, B_{n^{\prime}, \omega}\right]=0 .
$$

Эти коммутационные соотношения вместе с требованием $\left[B_{n, \omega}, H\right]=\omega B_{n, \omega}$ определяют коэффициенты $\alpha$ и $\beta$ (см. [3]):

$$
\begin{gathered}
\beta_{0 n}(\omega)=\frac{\omega-\omega_{n}}{\omega+\omega_{n}} \alpha_{0 n}(\omega) \\
\alpha_{1 n}\left(\omega, \omega^{\prime}\right)=\frac{\omega_{n}}{\omega+\omega_{n}} \alpha_{0 n}(\omega) V_{n}\left(\omega^{\prime}\right)\left[P \frac{1}{\omega-\omega^{\prime}}+y(\omega) \delta\left(\omega-\omega^{\prime}\right)\right] \\
\beta_{1 n}\left(\omega, \omega^{\prime}\right)=\frac{\omega_{n}}{\omega+\omega_{n}} \alpha_{0 n}(\omega) V_{n}\left(\omega^{\prime}\right) \frac{1}{\omega+\omega^{\prime}}
\end{gathered}
$$

где $\alpha_{0 n}(\omega)$ дается выражением

$$
\left|\alpha_{0 n}(\omega)\right|^{2}=\frac{\left(\omega+\omega_{n}\right)^{2}}{\omega_{n}^{2}} \frac{1}{V_{n}^{2}(\omega)\left[y_{n}^{2}(\omega)+\pi^{2}\right]},
$$

а $y_{n}(\omega)$ есть

$$
y_{n}(\omega)=\frac{1}{V_{n}^{2}(\omega)}\left[2 \frac{\omega^{2}-\omega_{n}^{2}}{\omega_{n}}+I_{n}(\omega)\right], \quad I_{n}(\omega)=P \int_{-\infty}^{+\infty} d \omega^{\prime} \frac{V_{n}^{2}\left(\omega^{\prime}\right)}{\omega^{\prime}-\omega} .
$$

В терминах новых переменных гамильтониан $H$ имеет вид

$$
H=\sum_{n} \int_{0}^{\infty} d \omega \omega B_{n, \omega}^{\dagger} B_{n, \omega}+E(a) .
$$


Здесь константа $E(a)$ имеет очевидный смысл энергии основного состояния системы, помимо изначального сдвига в (5). Отметим, что эта энергия была опущена в работе [3]. Изучение энергии основного состояния и ее компонент, порождаемых тремя частями гамильтониана, составляет основной предмет нашей работы.

Предполагая, что новые операторы $B_{n, \omega}$ и $B_{n, \omega}^{\dagger}$ образуют полный набор, можно обратить соотношения (9) и их сопряженные и выразить исходные операторы как линейные суперпозиции новых операторов:

$$
\begin{aligned}
b_{n} & =\int_{0}^{\infty} d \omega\left[\alpha_{0 n}^{*}(\omega) B_{n, \omega}-\beta_{0 n}(\omega) B_{n, \omega}^{\dagger}\right], \\
b_{n, \omega} & =\int_{0}^{\infty} d \omega^{\prime}\left[\alpha_{1}^{*}\left(\omega^{\prime}, \omega\right) B_{n, \omega^{\prime}}-\beta_{1}\left(\omega^{\prime}, \omega\right) B_{n, \omega^{\prime}}^{\dagger}\right] .
\end{aligned}
$$

Выражения (15) являются тем инструментом, который позволяет изучать энергию системы как сумму энергий квантового поля, среды и их взаимодействия, чему посвящен следующий раздел.

\section{3. ЭНЕРГИЯ ОСНОВНОГО СОСТОЯНИЯ Е И ЭНЕРГИЯ КАЗИМИРА}

Согласно формуле (14) основное состояние гамильтониана $H$ определяется условием $B_{n, \omega}|\rangle=0$. Его энергия, очевидно, есть $E$ в выражении (14). Вычисление $E$ не представляет труда. Используя (15), можно выразить старые операторы через $B_{n, \omega}, B_{n, \omega}^{\dagger}$ в исходном гамильтониане (5), а затем взять среднее в основном состоянии. Используя явные выражения для коэффициентов $\alpha_{0,1}$ и $\beta_{0,1}$, мы находим средние в основном состоянии:

$$
\begin{aligned}
& \left\langle H_{0}\right\rangle=\sum_{n} \omega_{n} \int_{0}^{\infty} d \omega \frac{\left(\omega-\omega_{n}\right)^{2}}{\left(\omega+\omega_{n}\right)^{2}}\left|\alpha_{0 n}(\omega)\right|^{2}, \\
& \left.\left\langle H_{\mathrm{I}}\right\rangle=2 \sum_{n} \omega_{n} \int_{0}^{\infty} d \omega \frac{\omega-\omega_{n}}{\omega+\omega_{n}} \mid \alpha_{0 n} \omega\right)\left.\right|^{2}, \\
& \left\langle H_{1}\right\rangle=\sum_{n} \omega_{n}^{2} \int_{0}^{\infty} d \omega \frac{1}{\left(\omega+\omega_{n}\right)^{2}} J_{n}(\omega)\left|\alpha_{0 n}(\omega)\right|^{2},
\end{aligned}
$$

где

$$
J_{n}(\omega)=\int_{0}^{\infty} d \omega^{\prime} \frac{\omega^{\prime} V_{n}^{2}\left(\omega^{\prime}\right)}{\left(\omega+\omega^{\prime}\right)^{2}} .
$$

Полная энергия основного состояния гамильтониана $H$ есть

$$
E(a)=\langle H\rangle=\left\langle H_{0}\right\rangle+\left\langle H_{1}\right\rangle+\left\langle H_{\mathrm{I}}\right\rangle .
$$

Полная энергия всей системы при заданном расстоянии между пластинами $a$ есть $E^{\text {tot }}(a)=E(a)+e_{0}(a)+e_{1}(a)$. Для нормировки мы должны вычесть из нее энергию вакуума в том же интервале без пластин. Она дается плотностью энергии вакуума, умноженной на $a$. Обозначим эту энергию $E^{\text {tot }}(\infty)$. Как и $E^{\text {tot }}(a)$, она состоит из трех частей: $E^{\text {tot }}(\infty)=E(\infty)+e_{0}(\infty)+e_{1}(\infty)$. Разность между $E^{\text {tot }}(a)$ и $E^{\text {tot }}(\infty)$ дает сумму двух энергий Казимира, для поля и для среды. Чтобы найти энергию 
Казимира для поля, мы должны дополнительно вычесть весь вклад в отсутствие поля, равный $\epsilon_{1}(a)-\epsilon_{1}(\infty)$. Таким образом, энергия Казимира поля есть

$$
E^{\mathrm{Cas}}=e_{0}(a)-e_{0}(\infty)+E(a)-E(\infty)=E_{0}^{\mathrm{Cas}}(a)+E_{1}^{\mathrm{Cas}}(a)
$$

где $E_{0}^{\text {Cas }}(a)=e_{0}(a)-e_{0}(\infty)$ есть стандартная энергия Казимира без наличия среды между пластинами, а $E_{1}^{\text {Cas }}=E(a)-E(\infty)$ есть дополнительная энергия, возникающая за счет взаимодействия с диэлектрической средой.

В отсутствие пластин наши выражения, полученные для конечных $a$, сохраняют свой вид, но только суммирование по дискретным значениям импульса $k_{n}$ заменяется на интегрирование:

$$
\sum_{n} \rightarrow a \int_{-\infty}^{+\infty} \frac{d k}{2 \pi}=a \int_{0}^{+\infty} \frac{d k}{\pi}=\int_{0}^{\infty} d n
$$

где мы использовали симметрию относительно замены $k \rightarrow-k$ и ввели непрерывную переменную $n$, полагая $k=\pi n / a$.

\section{4. ДИЭЛЕКТРИЧЕСКАЯ ПОСТОЯННАЯ И ПРОПАГАТОР}

Чтобы установить связь с диэлектрическими свойствами среды, рассмотрим нашу систему в отсутствие пластин. Запаздывающий пропагатор поля определяется как среднее в основном состоянии:

$$
P(t, x)=i \theta(t)\langle T[\phi(t, x), \phi(0,0)]\rangle, \quad \phi(t, x)=\int \frac{d k}{\sqrt{4 \pi \omega(k)}}(b(t, k)+\text { э.c. }),
$$

где $\omega(k)=k^{2}+\mu_{1}^{2}$. В диэлектрике операторы $b(t, k)$ и $b^{\dagger}(t, k)$, зависящие от времени, могут быть выражены через операторы $B(k, \omega)$, зависимость от времени которых тривиальна:

$$
b(t, k)=\int_{0}^{\infty} d \omega\left[\alpha_{0}^{*}(k, \omega) B(k, \omega) e^{-i \omega t}-\beta_{0}(k, \omega) B^{\dagger}(k, \omega) e^{i \omega t}\right]
$$

и аналогично для $b^{\dagger}(t, k)$. Новый вакуум удовлетворяет условию $B(k, \omega)|\rangle=0$. Выражая в $(19) \phi(t, x)$ через $B(k, \omega)$ и $B^{\dagger}(k, \omega)$ и переходя к энергетической переменной, мы получаем

$$
P(k, \omega)=\frac{1}{2 \omega(k)} \int_{0}^{\infty} d \omega^{\prime} \rho\left(k, \omega^{\prime}\right)\left(\frac{1}{\omega^{\prime}-\omega-i 0}+\frac{1}{\omega^{\prime}+\omega+i 0}\right),
$$

где

$$
\rho(k, \omega)=\left|\alpha_{0}(k, \omega)-\beta_{0}(k, \omega)\right|^{2} .
$$

На комплексной плоскости $\omega$ это определяет аналитическую функцию $\omega$ в верхней полуплоскости, удовлетворяющую условиям $P(-\omega *)=P^{*}(\omega)$ и

$$
\operatorname{Im} P(k, \omega)=\frac{\pi}{2 \omega(k)} \rho(\omega), \quad \omega>0 .
$$


Следующим шагом преобразуем $\alpha_{0}$ и $\beta_{0}$, следуя [3]. Используя (12) и (13), представим $\alpha_{0}(\omega)$ в виде

$$
\alpha_{0}(\omega)=\frac{1}{2}(\omega+\omega(k)) \frac{V(k, \omega)}{\epsilon^{*}(k, \omega) \omega^{2}-\omega_{0}^{2}(k)},
$$

где

$$
\epsilon^{*}(k, \omega)=1+\frac{\omega(k)}{2 \omega} F_{1}(k, \omega), \quad F_{1}(k, \omega)=\int_{-\infty}^{+\infty} \frac{d \omega^{\prime}}{\omega^{\prime}} \frac{V^{2}\left(k, \omega^{\prime}\right)}{\omega^{\prime}-\omega+i 0} .
$$

Вычисляя мнимую часть пропагатора согласно (23) и (22), мы находим, что он дается формулой

$$
P(k, \omega)=\frac{1}{\omega_{0}^{2}(k)-\omega^{2} \epsilon(k, \omega)},
$$

так что $\epsilon(k, \omega)$ имеет смысл диэлектрической постоянной.

\section{5. ПОДХОД ФУНКЦИИ ГРИНА}

Для сравнения с феноменологическим подходом, связанным с известной формулой Лифшица [9], следуя [7], вычислим энергию вакуума, используя феноменологическое уравнение и его функцию Грина. Согласно [7] такой подход ведет к результатам, соответствующим формуле Лифшица. Стандартные методы дают выражение для энергии поля в нашей модели

$$
E=\frac{1}{2} \sum_{n}\left(\dot{\phi}_{n}^{2}+\omega_{n}^{2} \phi_{n}^{2}\right)
$$

где $\omega_{n}^{2}=k_{n}^{2}+\mu^{2}$. Следуя [3], [7], введем диэлектрик через нетривиальную комплексную диэлектрическую постоянную $\epsilon(\omega)$ в уравнении движения для $\phi_{n}(\omega)$

$$
\left(\epsilon(\omega) \omega^{2}-\omega_{n}^{2}\right) \phi_{n}(\omega)=j_{n}(\omega)
$$

где $j_{n}(\omega)$ - "шум", ответственный за поглощение. Решение уравнения (28) может быть представлено в виде

$$
\phi_{n}(\omega)=\frac{j_{n}(\omega)}{\epsilon(\omega) \omega^{2}-\omega_{n}^{2}}
$$

Стандартным образом разделим $\phi_{n}(t)$ на части с положительными и отрицательными энергиями и будем их рассматривать как два набора независимых операторов, эрмитово сопряженных друг другу и определяемых источниками шума согласно формуле (29). Потребуем, чтобы они удовлетворяли стандартным коммутационным соотношениям. Можно показать, что для выполнения этого нужно выбрать [3]

$$
j_{n}^{( \pm)}(\omega)=\mp i \omega \sqrt{\frac{\epsilon_{2}}{\pi}} f_{n}^{( \pm)}(\omega),
$$

где $\epsilon_{2}=\operatorname{Im} \epsilon$ и операторы $f_{n}^{( \pm)}$имеют ненулевые коммутаторы

$$
\left[f_{n}^{(-)}(\omega), f_{m}^{(+)}\left(\omega^{\prime}\right)\right]=\delta_{m n} \delta\left(\omega-\omega^{\prime}\right) .
$$


Основное состояние определяется условием

$$
f_{n}^{(-)}(\omega)|\rangle=\phi_{n}^{(-)}(\omega)|\rangle=0 .
$$

Энергия основного состояния дается вакуумным средним (27). Выражая $\phi_{n}^{( \pm)}(\omega)$ сначала через $j^{( \pm)}(\omega)$, а затем через $f^{( \pm)}(\omega)$ и вычисляя вакуумное среднее с использованием (31) и (32), находим

$$
E(a)=-\frac{1}{2} \operatorname{Im} \sum_{n} \int_{0}^{\infty} \frac{d \omega}{\pi} \frac{\omega^{2}+\omega_{n}^{2}}{\epsilon(\omega) \omega^{2}-\omega_{n}^{2}} .
$$

Повернем контур интегрирования для следования вдоль мнимой оси $\omega=i \xi$, получим

$$
E(a)=\frac{1}{2 \pi} \sum_{n} \int_{0}^{\infty} d \xi \frac{\omega_{n}^{2}-\xi^{2}}{\epsilon(i \xi) \xi^{2}+\omega_{n}^{2}} \equiv \sum_{n} f_{n},
$$

где использовано, что функция $\epsilon(i \xi)$ вещественна.

При больших $\xi$ подынтегральное выражение в (34) стремится к -1 и интеграл становится независимым от $n$. Это позволяет добавить единицу к подынтегральному выражению. Нас интересует вклад в энергию Казимира за счет диэлектрика, который получается после вычитания из $E(a)$ ее значения при $\epsilon=1$.

Это дает окончательное выражение для энергии Казимира

$$
E_{1}^{\mathrm{Cas}}(a)=E(a)-E(a)_{\epsilon=1}=\sum_{n=1} f_{n}^{(1)}-\int_{0} d n f^{(1)}(n)
$$

где

$$
f_{n}^{(1)}=\frac{1}{2 \pi} \int_{0}^{\infty} d \xi\left(\frac{(\epsilon(i \xi)-1) \xi^{2}+2 \omega_{n}^{2}}{\epsilon(i \xi) \xi^{2}+\omega_{n}^{2}}-\frac{2 \omega_{n}^{2}}{\xi^{2}+\omega_{n}^{2}}\right)
$$

\section{6. ЧИСЛЕННЫЕ РАСЧЕТЫ}

Положим массу квантового поля равной нулю: $\mu=0$. Тогда вакуумная энергия Казимира есть $E_{0}^{\text {Cas }}=-\pi / 24 a$. Дополнительная энергия и сила, возникающая за счет взаимодействия с диэлектриком, в этом случае имеют форму

$$
E_{1}^{\text {Cas }}(a)=m e_{1}^{\text {Cas }}(m a), \quad F_{1}^{\text {Cas }}(a)=m^{2}\left[f_{1}^{\text {Cas }}(m a)\right]^{\prime},
$$

где $m$ - масштаб в $v(\omega)$.

В соответствии со свойствами $V_{n}(\omega)$ простейшая форма для $v^{2}(\omega)$ есть (вариант 1$)$

$$
v^{2}(\omega)=g^{2} \frac{1}{\omega^{2}+m^{2}}, \quad \epsilon(\omega)=1-\frac{\pi g^{2}}{2 m \omega} \frac{1}{\omega+i m},
$$

так что $\operatorname{Im} \epsilon(k, \omega)_{\omega \rightarrow 0} \sim 1 / \omega$. Чтобы включить в рассмотрение случай конечной $\operatorname{Im} \epsilon(k, \omega)$ при $\omega=0$, мы выбрали в качестве альтернативы (вариант 2)

$$
v^{2}(\omega)=g^{2} \frac{\omega^{2}}{\left(\omega^{2}+m^{2}\right)^{2}}, \quad \epsilon(\omega)=1-\frac{\pi g^{2}}{4 m(\omega+i m)^{2}}
$$

и $\operatorname{Im} \epsilon(k, \omega)=0$ при $\omega=0$. Мы положили $g^{2}=1 / \pi$. 


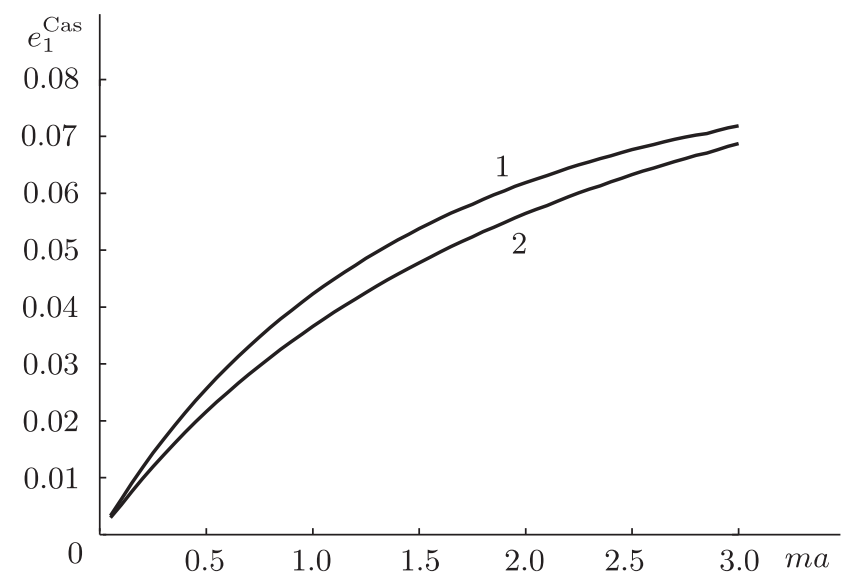

Рис. 1. Дополнительная энергия Казимира в диэлектрике $e_{1}^{\text {Cas }}(m a)$ для варианта 1 (37) (кривая 1) и варианта 2 (38) (кривая 2).

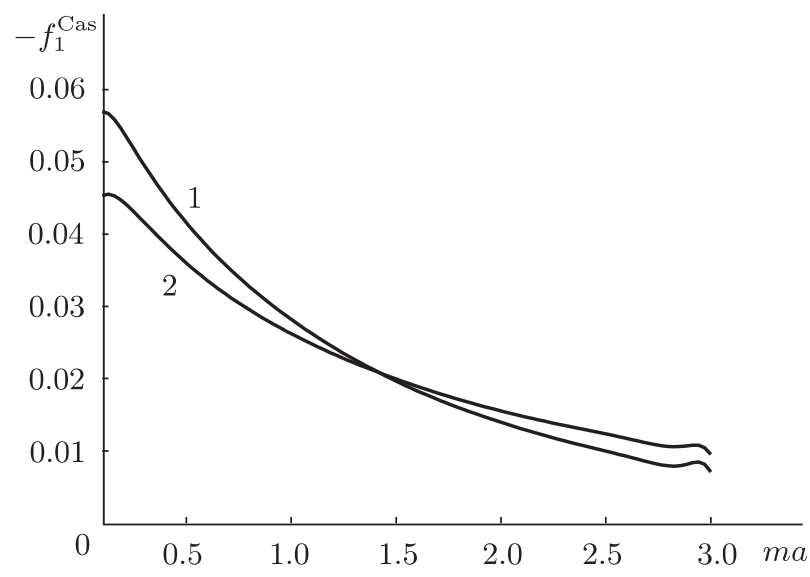

Рис. 2. Дополнительная сила Казимира в диэлектрике $f_{1}^{\mathrm{Cas}}(m a)$ для варианта 1 (37) (кривая 1) и варианта 2 (38) (кривая 2).

В обоих случаях интегралы $I_{n}$ и $J_{n}$ вычисляются аналитически и у нас остаются два численных интегрирования: по $\omega$ в выражениях для энергии при данном $\omega_{n}$ и при сравнении с $a \rightarrow \infty$.

Результаты численных расчетов энергии Казимира и силы в нашем подходе (формулы (16)) для обоих вариантов выбора $v^{2}$ представлены на рис. 1, 2. Как видно, дополнительная сила Казимира за счет взаимодействия с диэлектриком есть сила притяжения. Для выбранного значения $g^{2}$ она много меньше, чем вакуумная сила, в особенности при малых расстояниях $a$, где она остается конечной, тогда как вакуумная сила растет как $1 / a^{2}$.

Для сравнения мы также вычислили энергию Казимира в феноменологическом подходе, соответствующем формуле Лифшица (формулы (35) и (36)) для тех же вариантов выбора диэлектрической постоянной. Эти результаты для энергии и силы 


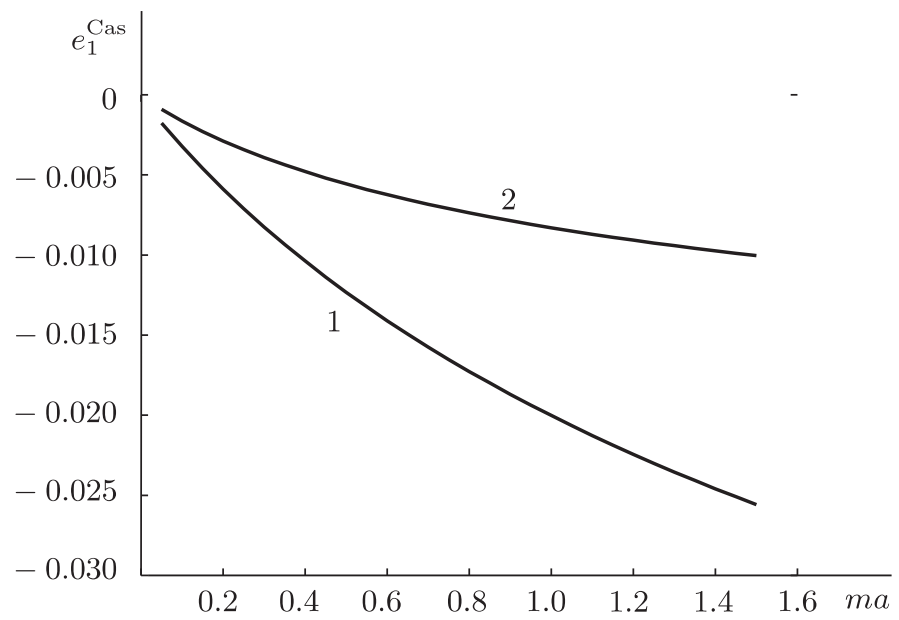

Рис. 3. Дополнительная энергия Казимира в диэлектрике $e_{1}^{\mathrm{Cas}}(\mathrm{ma})$ для варианта 1 (37) (кривая 1) и варианта 2 (38) (кривая 2) в феноменологическом подходе.

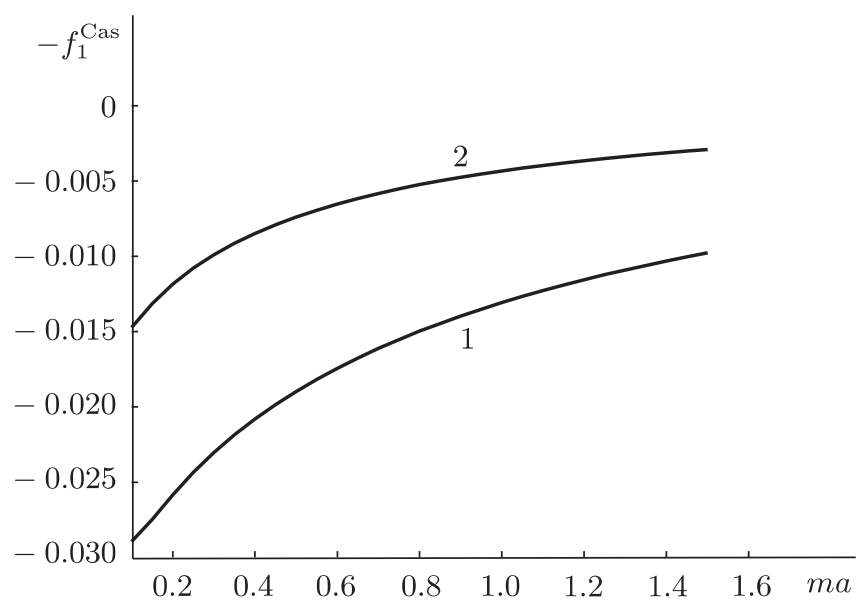

Рис. 4. Дополнительная сила Казимира в диэлектрике $f_{1}^{\mathrm{Cas}}(m a)$ для варианта 1 (37) (кривая 1) и варианта 2 (38) (кривая 2) в феноменологическом подходе.

Казимира показаны на рис. 3,4 для двух вариантов выбора $\epsilon$, соответствующих вариантам 1 и 2 выбора функции $v$.

\section{7. ЗАКЛЮЧЕНИЕ}

В нашей микроскопической картине, основанной на подходе, предложенном в работе [3], дополнительные энергия и сила Казимира оказываются малыми и соответствуют притяжению. Применение метода, основанного на феноменологической 
функции Грина, дает дополнительные энергию и силу того же порядка, но соответствующие отталкиванию. Подчеркнем, что последний подход не основывается на последовательной трактовке квантового поля и среды с их взаимодействием.

Благодарности. Настоящее исследование поддерживалось Санкт-Петербургским государственным университетом (проект № 11.0.77.2010).

\section{Список литературы}

[1] W. L. Mochán, C. Villarreal, New J. Phys., 8:10 (2006), 242, 22 pp.

[2] F. C. Lombardo, F. D. Mazzitelli, A. E. Rubio López, Phys. Rev. A, 84:5 (2012), 052517, $12 \mathrm{pp}$.

[3] B. Huttner, S. M. Barnett, Phys. Rev. A, 46:7 (1992), 4306-4322.

[4] T. Philbin, New J. Phys., 12 (2010), 123008, 22 pp.

[5] J. J. Hopfield, Phys. Rev., 112 (1958), 1555-1566.

[6] T. Philbin, New J. Phys., 13 (2011), 063026, 21 pp.

[7] C. Raabe, D.-G. Welsch, Phys. Rev. A, 71:1 (2005), 013814, 15 pp., arXiv: quant-ph/ 0408075.

[8] U. Fano, Phys. Rev., 124:6 (1961), 1866-1878.

[9] Е. М. Лифшиц, ЖЭТФ, 29:1 (1955), 94-110. 